\title{
Adaptive encoding of motion information in the fly visual system
}

\author{
Rafael Kurtz
}

\section{Contents}

Abstract 115

1. Introduction 115

2. Fly motion vision as a model for rapid sensory-motor control and for the transfer of biological principles to technical systems

3. Adaptation of sensory and neuronal systems to instantaneous stimulus levels . . . . . . . . 117

4. The computational principle of visual motion detection and its implications for the dynamic characteristics of neuronal motion signals 120

5. Motion adaptation changes the neuronal representation of image velocity and other stimulus parameters................
6. Stimulus-specific adaptation as a possible explanation for improved sensitivity to stimulus discontinuities . . . . . . . . . . 122

7. Interactions between different components of motion adaptation . . . . . . . . . . . . 123

8. Functional significance of adaptation in visual motion processing . . . . . . . . . . 124

Conclusions and outlook ................ 126

Acknowledgements. . . . . . . . . . . . . . 126

References.......................... 126

of adaptation in the fly motion vision system with simple stimulus paradigms as well as with complex, behaviorally generated stimuli is that adaptation improves the sensitivity for novel stimuli during exposure to sustained stimulation. Neuronal adaptation might thus facilitate important tasks such as object detection and obstacle avoidance during flight.

1.

\section{Introduction}

In biological systems visual motion plays an important role in the control of locomotion and navigation. Thus it is not surprising 
that principles of visual motion computation have been adopted from biology in the design of technical systems, e.g. autonomously navigating robots. One prominent example is flight control, in particular course stabilization and obstacle avoidance during flight. Control of flight movements presents an exceptionally challenging task for visual guidance. Compared to movement in a plane, greater degrees of freedom have to be handled and flight stabilization has to operate sufficiently quickly and reliably, in particular when ground crashes present a risk. However, the sensors involved in visual flight control are confronted with one major problem: the range of intensities and dynamics that have to be handled might often exceed a sensor's instantaneous operating range. Neuronal adaptation has been proposed to provide a valuable means to self-calibrate the system to the currently prevailing range of stimulus intensities. Moreover, adaptation might optimize the system's ability to extract from its complex continually varying input those stimulus features that are particularly relevant in the current behavioral situation. Adaptation-induced modifications of signal processing have been shown to be relevant in many sensory systems across a large range of model organisms (reviews: Menini 1999; Eatock 2000; Benda and Herz 2003; Fettiplace and Ricci 2003; Matthews and Reisert 2003; Kohn 2007; Wark et al. 2007). In this chapter visual motion processing in the fly brain is taken as an example in which the functional significance of adaptation can be studied in vivo by physiological experiments.
2.

\section{Fly motion vision as a model for rapid sensory-motor control and for the transfer of biological principles to technical systems}

The transfer of biological design principles to technical systems has been inspired by the in-depth study of sensory-motor control in several biological model systems (review: Webb 2002). One of these biological models turned out to be of exceptional value: visual motion-processing in flies (in particular the fruit fly Drosophila, blowflies and hoverflies). The significance of the fly as a model system for rapid sensory-motor control is due to a number of advantages. In flies, it is feasible to combine the analysis of visually guided locomotor behavior with the investigation of the neuronal architecture and the neuronal computations underlying this behavior (reviews: Egelhaaf et al. 2005; Egelhaaf 2008; Borst 2009). Unlike in many other model systems, in flies there appear to be comparatively few processing steps involved in the extraction of specific motion information from visual cues. Moreover, a prominent class of neurons, consisting of less than 100 cells per brain hemisphere, converts visual motion information into neuronal signals suitable for visually guided motor control. This class of neurons, the tangential cells (TCS) of the lobula plate, has been particularly well studied in the blowfly Calliphora vicina (Fig. 1A; reviews: Borst and Haag 2002; Egelhaaf et al. 2005). Particular TCs have distinct morphological and functional properties, making them individually identifiable in electrophysiological recording experiments (reviews: Hausen and Egelhaaf 1989; Borst and Haag 2002) and in functional imaging studies (review: Kurtz et al. 2008).

TCs perform a crucial step in the transfer of visual motion input into output signals for locomotor control: they integrate local mo- 
A

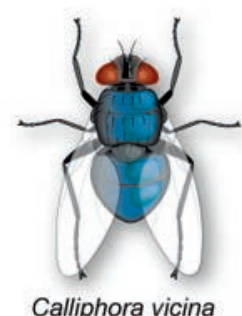

$\mathrm{C}$

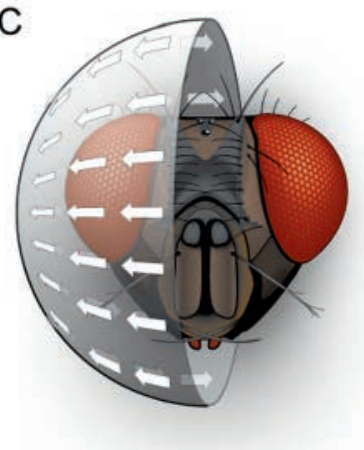

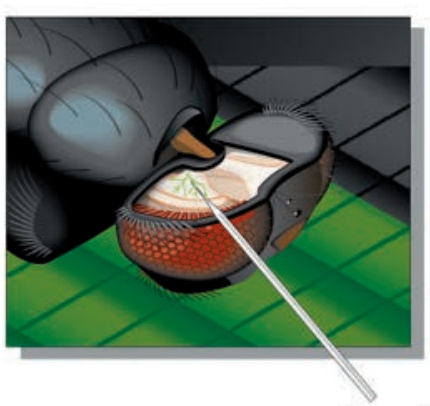

B

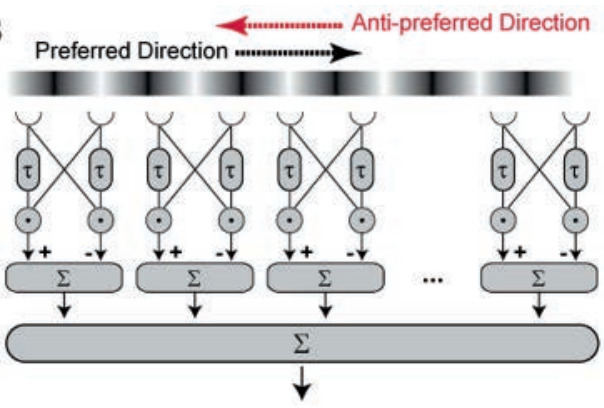

Recording of membrane potential of TCs during motion stimulation

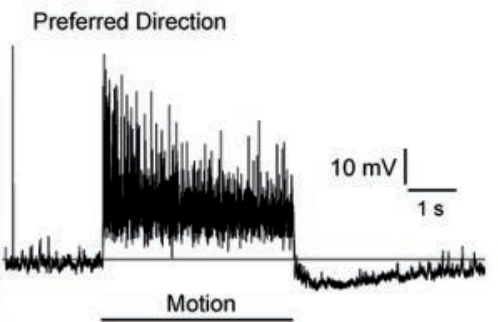

Fig. 1 Neuronal processing of visual motion in the fly brain. A, Preparation of Calliphora vicina (left) used to record from TCs in the third visual neuropil, the lobula plate (right). B, Computational principle thought to underlie motion computation. Motion is first computed by elementary motion detectors (EMDs), which correlate (by multiplication, ".") the brightness signal from one location in the visual field with a temporally delayed (by a low pass filter with time constant $\tau$ ) brightness signal from a neighboring location. Subtraction of the output from one detector half unit from its mirror-symmetric counterpart yields a local motion signal that is positive for motion in preferred direction and negative for motion in anti-preferred direction. Integration over an array of EMDs provides global motion signals. TCs integrate at their dendrites the outputs from large 2-dimensional arrays of retinotopically arranged EMDs. C, Motion response of a TC, consisting of a pronounced graded change of the axonal membrane potential and a change in the frequency of action-potential-like transients with variable amplitude ("spikelets"). Horizontal line indicates resting potential

tion inputs over large parts of the visual field (Krapp et al. 1998; Spalthoff et al. 2010). Reliable information about global motion patterns is extracted from retinal image shifts during self-motion (Krapp and Hengstenberg 1996). Thus, fly TCs are likely to play a prominent role in providing the motor system with the specific sensory signals that are required for visually guided flight stabilization and course control (see also Chapter II,5 by Krapp and Taylor).
3.

\section{Adaptation of sensory and neuronal systems to instantaneous stimulus levels}

In biological as well as in technical systems, sensors face one common problem: The wide range of intensities over which their input signal may modulate is contrasted with a limited range of possible output states. Severe constraints to the working range of 
sensory cells and neurons may result directly from the reversal potentials of the excitatory and inhibitory ionic currents or from a limit in spike rate. Corresponding working range limitations in technical systems are, for example, the finite capacity of photon conversions on a CCD chip or the mechanical displacement limit of an airflow sensor. The working range of biological as well as technical systems can be defined as the range over which a change in input intensity results into a sizable change of the output signal. In principle, the working range of a sensor could be broadened even in the presence of a fixed output limit by decreasing the system's gain, i. e. the slope of the input-output function. Unfortunately, this simple strategy is problematic because a lower slope of the input-output function inevitably results into weaker input-driven modulations of the output. Weak output modulations are, however, much more prone to corruption by downstream noise, in particular when fast modulations of the input signal need to be encoded, as is the case in visual motion processing.

Instead of reducing the slope of the inputoutput function, the entire function may be shifted horizontally to a certain range of input intensities, without changing the shape of the function. If such shifts become effective in a stimulus-history dependent way, the capability of a sensor to encode a large spectrum of possible input intensities can be improved without sacrificing the precision of output signals (Fig. $2 \mathrm{~A}$ ). In this way the system can take advantage of the fact that in most natural input signals characteristic temporal correlations are present, and use a strategy of "predictive coding" (see Srinivasan et al. 1982 for similar considerations concerning spatial visual coding). For example, the probability that luminance changes abruptly from bright sunlight to pitch dark is usually very low during natural visual stimulation. Thus the mean stimulus intensity in a certain time window of stimulus history often provides a good estimate of near future stimulus intensities. Therefore it might be useful to adjust a sensor's input-output relationship according to this estimate, thereby enhancing sensitivity to changes in the current stimulus intensity. When schematized as a single sigmoid inputoutput function a theoretically plausible adaptation-induced modification would shift the function such that the range of current stimulus intensities is optimally covered by the high-slope region of the curve (Fig. 2A).

In the fly motion vision system a large variety of different adaptation phenomena has been demonstrated over the past three decades (Srinivasan and Dvorak 1979; Maddess and Laughlin 1985; de Ruyter van Steveninck RR et al. 1986; Brenner et al. 2000; Harris et al. 2000; Kurtz et al. 2000; Fairhall et al. 2001; Reisenman et al. 2003; Borst et al. 2005; Heitwerth et al. 2005; Neri and Laughlin 2005; Kurtz 2007; Neri 2007; Kalb et al. 2008a; Kalb et al. 2008b; Liang et al. 2008; Kurtz et al. 2009a; Kurtz et al. 2009b; Nordström and O'Carroll 2009). In the following I will first outline the computational principle by which motion information is thought to be extracted from the visual stimulus. I will then describe some of the adaptation phenomena observed in visual motion-processing and examine the extent to which simple adaptation models (see Fig. 2) may help us to understand these phenomena. Finally, I will address the putative functional significance of motion adaptation under real-life conditions. 

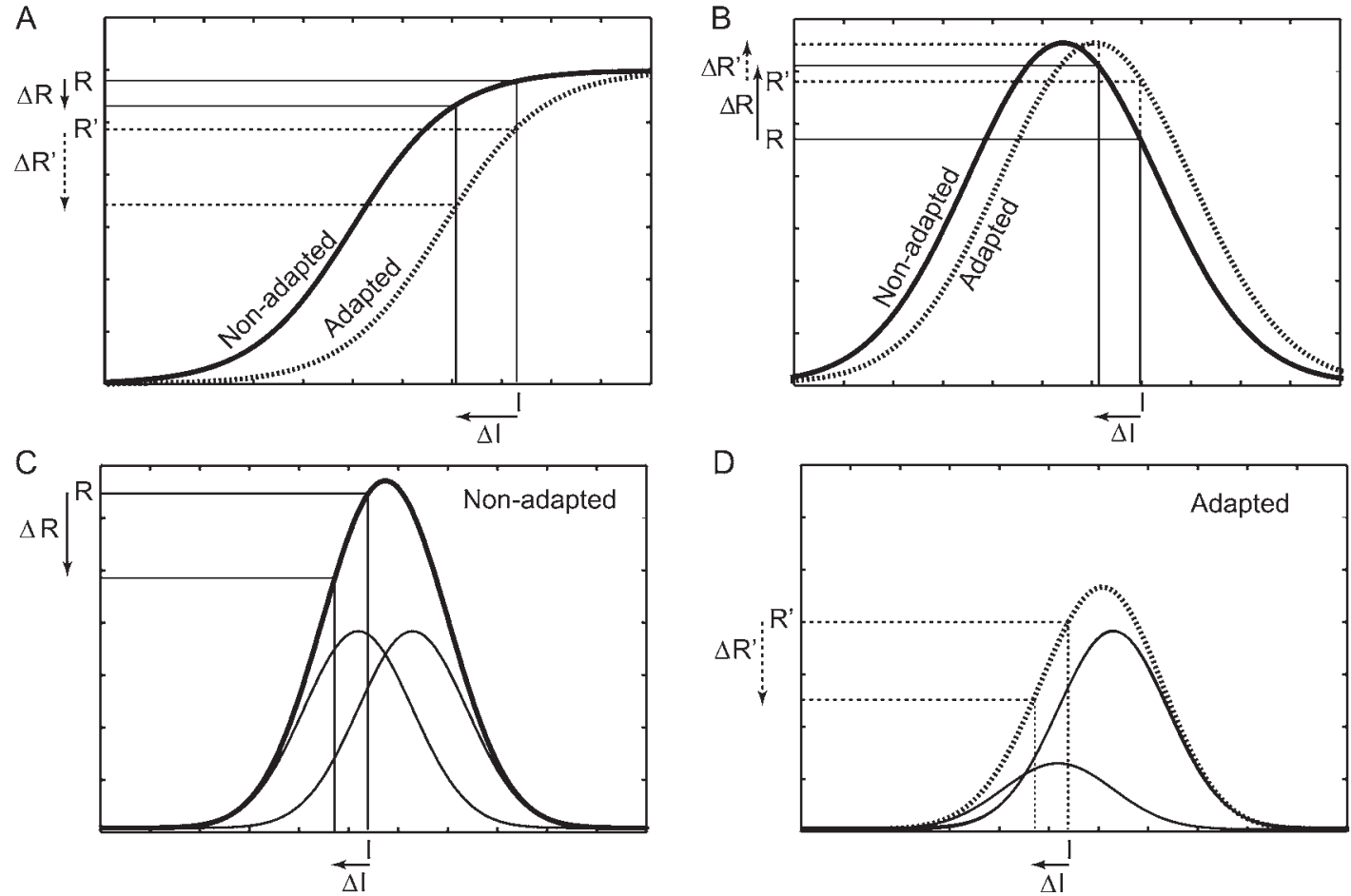

Fig. 2 Possible adaptation-induced changes of input-output functions. A, Sigmoid input-output function, as is characteristic for the stimulus-response relationship of many neurons and sensory. Sustained high input intensity (I) causes adaptation, resulting in a lateral shift of the function towards higher input intensities. The shift causes attenuation of steady-state responses ( $R^{\prime}$ vs $R$ ) and enhanced responses $\left(\Delta R^{\prime}\right.$ vs $\Delta R$ ) to changes in input intensity $(\Delta \mathrm{I})$. B, Input-output relationships may also follow bell-shaped functions, for example responses of visual motion-sensitive neurons to different velocities. Adaptation-induced shifts similar to those in $A$ would enhance $\Delta \mathrm{R}$ only in that part of the curve which has a positive slope. In contrast, as illustrated in the example, for inputs falling on parts of the curve with negative slope adaptation would lead to an increase of the steady-state response ( $R^{\prime}$ vs $R$ ) and to a decrease of the response to changes in input intensity ( $\left.\Delta R^{\prime} v s \Delta R\right)$. C, D, A bell-shaped input-output function (thick lines) is depicted, which results from integration of two inputs with peaks at different stimulus intensities (thin lines). Adaptation is assumed here to cause a simple attenuation of inputs (instead of a lateral shift as in A and B). This attenuation is "stimulus-specific", affecting only the input that shows the highest activity at the given stimulus level (I). This form of adaptation leads to a decrease in the steady-state value of the integrated response ( $R^{\prime}$ vs $R$ ), but to nearly unaltered responses to changes in input intensity $\left(\Delta \mathrm{R}^{\prime}\right.$ vs $\left.\Delta \mathrm{R}\right)$. Thus, the relative sensitivity to changes in input intensity $(\Delta \mathrm{R}$ relative to $\mathrm{R}$ or, respectively, $\Delta \mathrm{R}^{\prime}$ relative to $\mathrm{R}^{\prime}$ ) is enhanced with adaptation 
4.

\section{The computational principle of visual motion detection and its implications for the dynamic characteristics of neuronal motion signals}

During movement of an animal's or human's eyes, head or entire body, its retinas experience a continual image displacement. In a wide range of animal species, ranging from insects to monkeys, such global motion is processed by neurons that sample local motion across the visual field in a retinotopic way (reviews: Krapp 2000; Lappe 2000). In flies, this type of neuron is represented by the class of TCs (see above; reviews: Hausen and Egelhaaf 1989; Borst and Haag 2002; Egelhaaf et al. 2005). The responses of these neurons, however, depend not only on image velocity, but also on the contrast, the spatial frequency content and the orientation of pattern elements. Thus it is not surprising that adaptation of TCs has been shown to alter responsivity not only for velocity, but also for other features that characterize the motion stimulus (see sections 5-7).

As in other species, the local motiondetecting elements, which supply input to fly TCs, have so far largely evaded a direct experimental investigation. However, plausible computational models of local motion detection have been inferred based on the properties of TCs. One such model is the correlation-type motion detector, often referred to as the elementary motion detector (EMD) (review: Borst and Egelhaaf 1989). EMDs are based on correlating the appropriately filtered brightness signals from neighboring points in visual space (Fig. 1B). It has been shown that EMDs can explain the responses of motion-sensitive neurons to a wide range of motion stimuli (Egelhaaf and Reichardt 1987; Lindemann et al. 2005).

EMDs have several distinguishing features that are relevant in the context of motion adaptation. Most importantly, EMDs are not veridical sensors of local retinal velocities. (1) The time-averaged response to constant velocity motion increases with pattern velocity only within a certain velocity range; it then reaches an optimum and decreases again (Egelhaaf and Borst 1989). (2) The responses of individual EMDs modulate over time depending on the local pattern features within their receptive fields (Egelhaaf et al. 1989). (3) These modulations can be eliminated to some extent by spatial integration (Egelhaaf and Borst 1989). However, even spatially pooled EMD outputs have a characteristic phasic-tonic time course during stimulation with constant velocity (see Fig. 1C, 3). This temporal response profile is a direct consequence of the computational principle of EMDs and is thus manifested without an activity-dependent change in a system parameter, i. e. adaptation. Intriguingly, it has been shown that even more complex changes in response properties are emergent properties of the EMD. In particular, both in recordings from TCs as well as in EMD models with fixed parameters, it was observed that the slope of the neuronal input-output function changes when random velocity fluctuations of different modulation depths are presented (Borst et al. 2005). This phenomenon was termed "adaptation without parameter change" to differentiate it from "adaptation" in a strict sense, for which genuine changes in system parameters have to be present. In this context it is important to mention that for most of the adaptation phenomena described in the following it is at present not clear whether they result from physiological changes in the TCs themselves or from the properties of neurons at earlier stages of the visual pathway. 
5.

\section{Motion adaptation changes the neuronal representation of image velocity and other stimulus parameters}

In fly TCs, motion adaptation was first demonstrated in the H1-neuron (Maddess and Laughlin 1985). During adaptation with sustained pattern motion in preferred direction the response of $\mathrm{H} 1$ decreased. Nonetheless, the response transients elicited by velocity discontinuities, i. e. brief increments or decrements from baseline velocity, became more pronounced in the course of adaptation (see also Fig. 3A). This result is in accordance with the view that adaptation improves neuronal sensitivity to changes in stimulus intensity around the current level. The mechanism underlying this phenomenon was proposed to be an activity-dependent down regulation of the time constant of the EMD low-pass filter. This parameter change would shift velocity tuning towards higher values (de Ruyter van Steveninck RR et al. 1986; Clifford and Langley 1996; Clifford et al. 1997). However, with such a mechanism alone, an enhancement of response transients to velocity discontinuities by motion adaptation would be restricted to low velocities. At high velocities the response transients evoked by changes in velocity might even become weaker in the adapted than in the non-adapted state. This prediction can be made with regard to the bell-shaped signature of velocity tuning curves (Fig. 2B), but was not experimentally tested (Maddess and Laughlin 1985). Therefore, recently the question was addressed, whether enhanced sensitivity to velocity changes in the course of motion adaptation is also present at high baseline velocities (Kurtz et al. 2009b). It was found that the transient deflections in spike rate of $\mathrm{H} 1$ in response to discontinuities in motion velocity were enhanced by

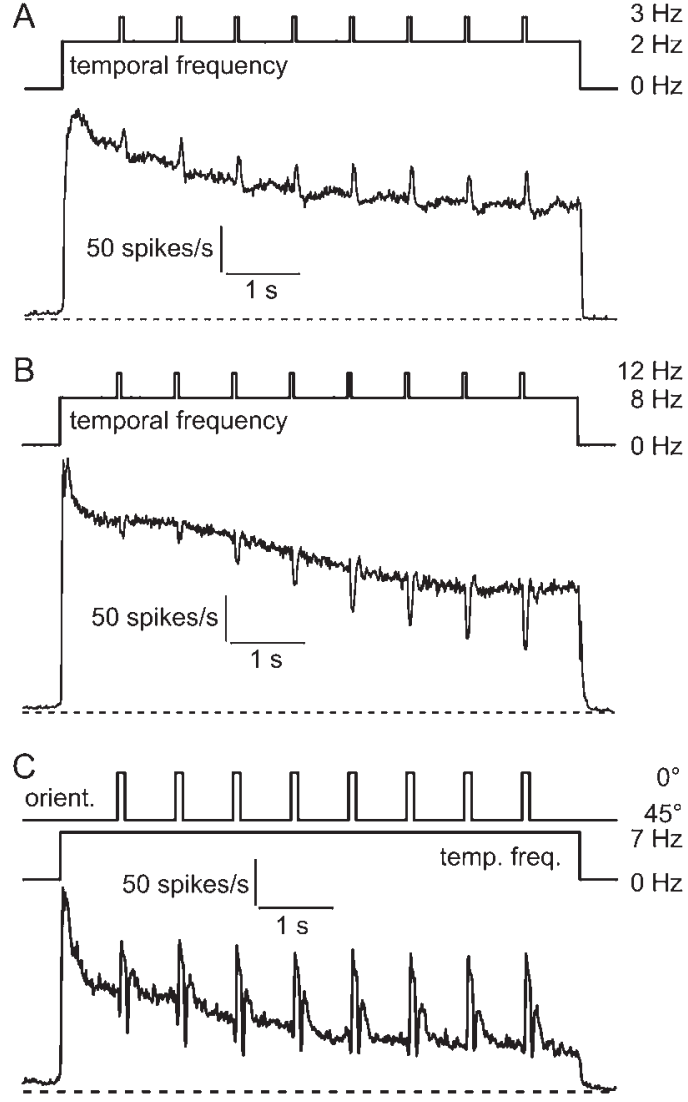

Fig. 3 Enhancement of responses to sudden stimulus changes with motion adaptation. A, Spike rate of a Calliphora $\mathrm{H} 1$ neuron during motion of a periodic grating at constant velocity interspersed with brief increases in velocity. Although the neuronal response to the baseline velocity decreases with adaptation, the response to velocity increments is enhanced. The values in $\mathrm{Hz}$ indicate temporal frequency of the grating, which is linearly related to velocity. B, Data obtained from a similar experiment but with baseline velocity above the steady-state optimum of the $\mathrm{H} 1$ neuron. Under this condition, increments in motion velocity lead to decrements in spike rate. Nevertheless, the amplitude of these decrements increases with adaptation. $\mathbf{C}$, Discontinuities in the motion stimulus produced by changing the orientation of the grating, and thus the direction of motion $\left(0^{\circ}=\right.$ preferred direction). Again, adaptation enhances responses to the stimulus discontinuities. Modified from Kurtz et al. 2009b 
adaptation at baseline velocities below as well as above the steady-state velocity optimum (Fig. 3A,B). This finding indicates that the hypothetical adaptation-induced shift of the neuronal velocity tuning towards higher values cannot entirely account for the enhancement of response transients to velocity discontinuities. In accordance with this result, consistent shifts of velocity tuning were neither found after adaptation with constant-velocity motion (Harris et al. 1999) nor after random velocity modulations (Kalb et al. 2008b). Next, it was tested whether a similar enhancement of response transients with motion adaptation is also present when the discontinuity in the visual motion stimulus is not produced by a change in velocity, but in one of the other stimulus parameters. Intriguingly, it was found that responses to discontinuities in motion direction (Fig. 3C) as well as in the contrast or the wavelength of a moving grating were also accentuated in the course of motion adaptation (Kurtz et al. 2009 b). These findings suggest that adaptation improves neuronal sensitivity to sudden changes in any of the parameters of the motion stimulus.

6.

\section{Stimulus-specific adaptation as a possible explanation for improved sensitivity to stimulus discontinuities}

A phenomenon, stimulus-specific adaptation has been proposed to explain why many neurons are able to maintain their sensitivity to changes in the stimulus while their responses during sustained uniform stimulation are strongly attenuated (Ulanovsky et al. 2003). Stimulus-specific adaptation provides a concept that might explain an increased sensitivity to stimulus discontinuities by adaptation without assuming a genuine shift in the stimulus-response function. Consider a neuron that integrates inputs from several elements that differ in their response optima (Fig. 2C). Adaptation with sustained stimulation activates most strongly those input elements which have their optimum close to the adapting stimulus. However, due to the shallow slope of their response function close to the peak, these inputs would show only small changes in activity in response to a stimulus discontinuity, i.e. to transient increases or decreases in stimulus intensity. A simple activity-dependent attenuation of these highly activated inputs would then improve the relative sensitivity to stimulus discontinuities. This is the case because after attenuation of strongly activated inputs the inputs that are only moderately activated by the adapting stimulus would have a relatively stronger impact on the postsynaptic integrating neuron. The overall response to stimulus discontinuities would then be enhanced relative to the background response, because the moderately activated inputs, operating in a high-slope regime of their stimulus-response function, show strong responses to stimulus changes (Fig. 2D).

Stimulus-specific adaptation has been implicated in processes that enable neurons to function as efficient 'novelty detectors', with the ability to extract the appearance of new stimulus features during sustained stimulation. For example, in the auditory system, frequency-specific adaptation is thought to facilitate the detection of novel sounds in the presence of a sustained stimulation by sound mixtures (Ulanovsky et al. 2003; Reches and Gutfreund 2008). The ability of a neuronal system to adapt in a stimulus-specific manner depends on the distinct representation of different stimulus qualities or features by individual input elements. In visual motion processing this important prerequisite for stimulus-specific adapta- 
tion is at least partially met. In particular, many TCs receive input from neurons that differ in their preferred motion directions (Krapp et al. 1998; Spalthoff et al. 2010). If these inputs are attenuated during ongoing stimulation with motion, it is likely that this attenuation is strongest in those inputs that most closely match their preferred direction to the adapting motion direction. Analogous to the scheme in Fig. 2C,D, specific attenuation of strongly activated inputs could improve the sensitivity to changes in motion direction. Although it is intriguing to propose stimulus-specific adaptation as a putative mechanism underlying the enhanced sensitivity for changes in motion direction (Fig. 3C), the corresponding changes in the activity of inputs are still to be demonstrated directly in fly TCs.

Is stimulus-specific adaptation also a plausible explanation for increased sensitivity of fly TCs to discontinuities in motion velocity (Fig. 3A,B)? This form of adaptation would require inputs that differ in their velocity optimum, and are thus differentially activated by a given baseline velocity and, consequently, attenuated to different degrees during adaptation. As systematic recordings from the inputs of fly TCs are hard to obtain, differences in velocity tuning of inputs can only be indirectly deduced from the existence of multiple peaks in the velocity tuning of TCs. Whereas the velocity tuning of fly TCs appears to be smooth, distinct peaks at different velocities were shown to be present in the velocity tuning of motion-sensitive neurons of other insect species (O'Carroll et al. 1996; O'Carroll et al. 1997). Apart from enabling stimulus-specific adaptation, the coexistence of EMDs with different velocity tuning in the visual system would present a valuable prerequisite for extracting velocity independent of spatial pattern properties (Srinivasan et al. 1999).
7.

\section{Interactions between different components of motion adaptation}

In the previous section, the issue of how motion adaptation changes the sensitivity to modulations in one of the stimulus parameters was addressed. However, the effects of motion adaptation on the signaling of different stimulus parameters interact with one another. For example, as will be outlined below, pronounced changes in contrast sensitivity are induced by motion adaptation, and these may in turn have a strong impact on direction sensitivity.

Harris et al. (2000) analyzed in detail how motion adaptation alters contrast sensitivity of one class of TCs, Horizontal-System (HS) neurons of the hoverfly Eristalis tenax. Unlike the velocity-response function or the direction tuning, which form bell-shaped or sinusoidal curves, respectively, the contrastresponse function forms a sigmoid. Thus, contrast coding would profit over the entire range of contrasts from an adaptive shift of the curve's region of highest slope towards the mean contrast level of the present stimuli (as shown in Fig. 2B). A strong shift of the contrast-response function towards higher contrasts was indeed found to be present after adapting the neurons with motion of a high-contrast grating (Fig.4). This rightward shift was not present when adapting and test stimuli were presented in different, non-overlapping regions of the large receptive field of HS-neurons (Nordström and O'Carroll 2009). This property implies that the underlying cellular adaptation process is not generated in TCs themselves, but at a more peripheral location.

In addition to the rightward shift of the contrast-response function, two further adaptation components contributed to the attenuation of contrast sensitivity: a subtractive shift of the contrast-response function 

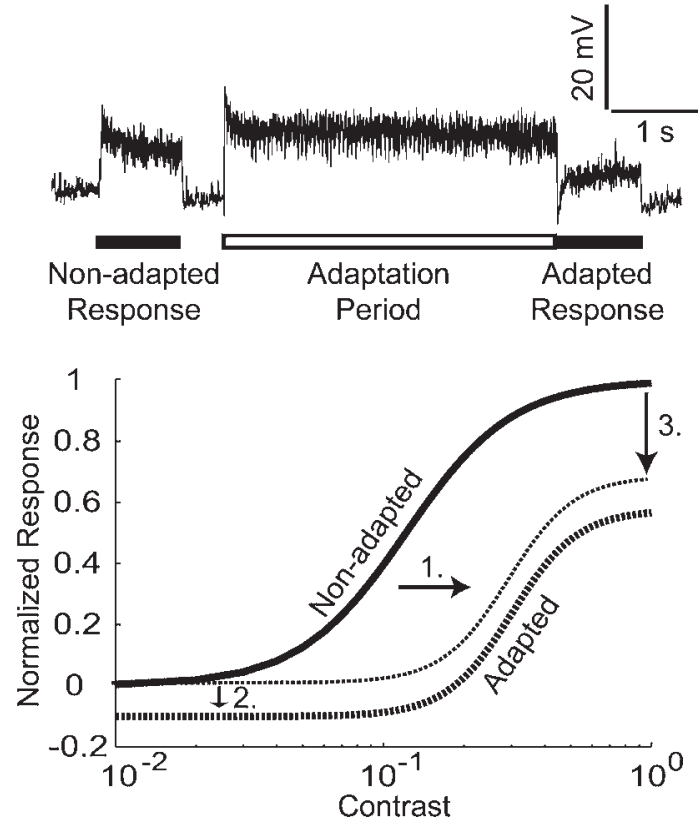

Fig. 4 Motion adaptation affects contrast gain. Top, response of an HS neuron of the hoverfly Eristalis tenax to a drifting grating of medium luminance contrast measured before and after adaptation with motion of a high-contrast grating. Bottom, schematic of contrast-response curves obtained by measuring non-adapted and adapted responses to drifting gratings of various contrasts. Three components of motion adaptation contribute to the attenuation of contrast sensitivity: 1) lateral shift towards higher contrast values; 2 ) downward shift, equivalent to neuronal after-hyperpolarization. 3) compression of the output range, which is best visible when the adapted curve is corrected for the afterhyperpolarization (thin dotted line). Modified from Harris et al. (2000)

and a compression of the output range of the neuron (Fig.4). Whereas the origin of the latter phenomenon is unknown, the subtractive shift is caused by a depolarizationactivated conductance, which manifests itself as a hyperpolarization after stimulus offset (see also Fig. 1C). This after-hyperpolarization might form the neural substrate of the famous "waterfall illusion" derived from human psychophysics, in which prolonged viewing of a strong motion stimulus induces the strong impression of motion in the opposite direction when a stationary pattern is fixated afterwards (review: Anstis et al. 1998). Interestingly, a behavioral correlate of the "waterfall illusion" has been observed in the fly's optomotor turning response (Srinivasan and Dvorak 1979). The cellular mechanism underlying the after-hyperpolarization is a depolarization-activated conductance of the TCs (Kurtz 2007; Kurtz et al. 2009a).

All the adaptation components mentioned above affect the responses of the neuron to subsequently presented motion independent of its direction. Intriguingly, adaptation of contrast sensitivity might not only modify contrast coding itself, but also direction selectivity. Changes in direction selectivity with adaptation were demonstrated in the V1-neuron of Calliphora (Kalb et al. 2008a). This TC prefers vertical motion, but also responds weakly to horizontal motion. Following adaptation with either vertical or horizontal motion, the ratio of vertical motion responses versus horizontal motion responses was increased in V1. A parsimonious explanation for this finding is that the responses to weak stimuli, for example, motion in a direction that differs from the neuron's preferred direction, are more affected by a subtractive shift of the contrastresponse function than responses to strong stimuli.

\section{8. \\ Functional significance of adaptation in visual motion processing}

As detailed above, motion adaptation affects directional tuning as well as contrast sensitivity of fly TCs, and it leads to enhanced 

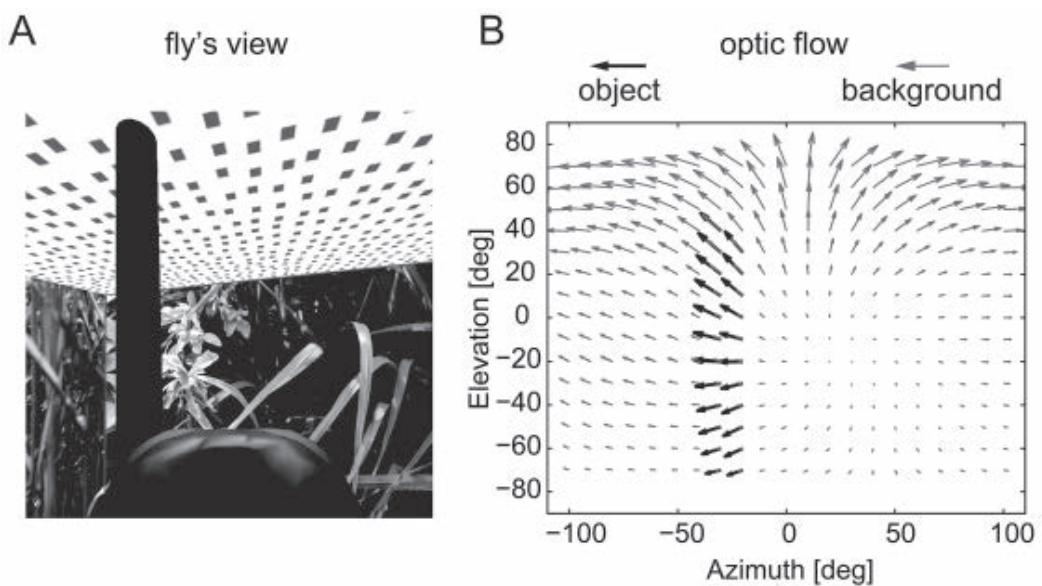

\section{C with object}
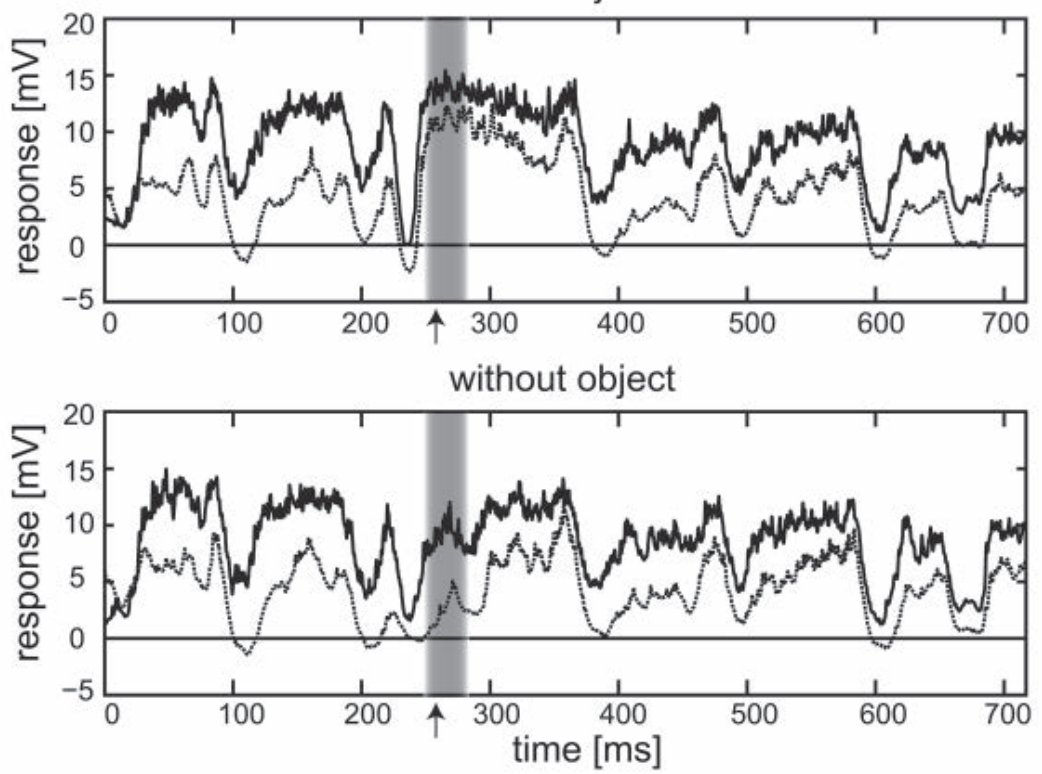

Fig. 5 Motion adaptation accentuates responses to objects in a three-dimensional environment. A, Virtual environment presented to the fly (part of the head sketched at the bottom) in neuronal recording experiments. Trajectories and head orientations of Calliphora during flights in a cubic arena with patterned walls were monitored. Visual stimuli encountered by the fly were calculated and replayed on a high-speed panoramic visual stimulator during electrical recording of HS-neurons. Responses to the original image sequence were compared with those in which a virtual object (dark bar in the left half of the image) was placed close to the fly's trajectory. B, Visual motion experienced by the fly at the instant of time shown in A. Arrows represent velocity vectors at different points in visual space. The object induces distinct motion cues during forward translation of the fly because it is closer to the fly than the background. C, Average neuronal responses of 10 HS-neurons in non-adapted (solid line) and adapted state (dotted line) to image sequences with object (top) and without object (bottom). Grey shading indicates an interval during which the object is in the receptive field of the HS neuron (and the corresponding interval in the "without object" condition). Arrows indicate moment shown in A. In general, the neuronal response is attenuated with adaptation. However, the attenuation is much weaker when the object is in the receptive field of the neuron (upper plot, shaded area). Modified from Liang et al. (2008) 
sensitivity for various types of stimulus discontinuities (Fig. 3). Adaptation might thus facilitate the extraction of those features of the stimulus that are relevant in a given behavioral situation. This view is supported by a recent study, in which Calliphora HS-cells were stimulated with naturalistic visual motion (Fig. 5A,B; Liang et al. 2008). A strong overall reduction in the neuronal response was observed after persistent stimulation. However, this adaptation-induced attenuation was weaker in time segments during which a virtual object moved into the receptive field (Fig. 5C). Thus, motion adaptation appears to enhance the ability of the neuron to contribute to the detection of novel signals (exemplified by the virtual object), which are potentially more important than continuing, unchanged signals.

A further benefit of adaptation may result directly from the strong decrease in overall activity and the associated decrease in energy demand. Local energy availability in a brain area may be a constraint, because neuronal signaling involves metabolically costly processes such as action potential propagation and synaptic transmission (Laughlin 2001). It has been shown that, consistent with this idea, $\mathrm{H} 1$ decreases its spike rate during adaptation by naturalistic stimulation (Heitwerth et al. 2005). Importantly, the information content of $\mathrm{H} 1$ spike trains was not reduced proportionally, resulting in considerably more information per spike.

\section{Conclusions and outlook}

At the current level of knowledge there appears to be a discrepancy between the successful phenomenological investigation of adaptation in visual motion processing neurons and the assessment of the functional significance of these phenomena for visually guided motor control in natural conditions. On the one hand, the number of different adaptation phenomena in fly motion vision described over the past 25 years is so large that only a few of them could be considered in the present chapter. On the other hand, systematic investigations of the specific roles of different components of motion adaptation in the processing of natural stimuli are still lacking.

Putative functional implications of motion adaptation, such as enhanced object detection (Liang et al. 2008), depend on how efficiently different cellular adaptation processes are activated by the complex temporal profile of a natural visual motion stimulus. Since the visual input is shaped by the fly's own movements, the resulting visual motion stimulus can have dramatically diverse dynamic characteristics (Kern et al. 2001; Boeddeker et al. 2003; Kern et al. 2005). Thus it is plausible to assume that an efficient extraction of behaviorally relevant environmental features depends on whether adaption mechanisms and its time constants are adjusted to the temporal statistics of input signals. It has been concluded that adaptation of fly motion-sensitive neurons to the statistics of random velocity modulations of a grating pattern improves the extraction of velocity information (Brenner et al. 2000). One major future task is to analyze the dynamics of visual motion occurring in natural flight situations and to test how far these temporal characteristics are matched by the dynamic properties of adaptation.

\section{Acknowledgements}

The author's labwork was supported by the Deutsche Forschungsgemeinschaft (DFG). C. Spalthoff contributed to the design of figure 1.

\section{References}

Anstis S, Verstraten FA, Mather G (1998) The motion aftereffect. Trends Cogn Sci 2: 111-117

Benda J, Herz AV (2003) A universal model for spike-frequency adaptation. Neural Comput 15: 2523-2564

Boeddeker N, Kern R, Egelhaaf M (2003) Chasing a dummy target: smooth pursuit and velocity control in male blowflies. Proc R Soc Lond B Biol Sci 270: 393-399

Borst A (2009) Drosophila's view on insect vision. Curr Biol 19: R36-R47 
Borst A, Egelhaaf M (1989) Principles of visual motion detection. Trends Neurosci 12: 297-306

Borst A, Flanagin VL, Sompolinsky H (2005) Adaptation without parameter change: Dynamic gain control in motion detection. Proc Natl Acad Sci U S A 102: 6172-6176

Borst A, Haag J (2002) Neural networks in the cockpit of the fly. J Comp Physiol [A] 188: 419-437

Brenner N, Bialek W, de Ruyter van Steveninck RR (2000) Adaptive rescaling maximizes information transmission. Neuron 26: 695-702

Clifford CW, Ibbotson MR, Langley K (1997) An adaptive Reichardt detector model of motion adaptation in insects and mammals. Vis Neurosci 14: 741-749

Clifford CW, Langley K (1996) Psychophysics of motion adaptation parallels insect electrophysiology. Curr Biol 6: 1340-1342

de Ruyter van Steveninck RR, Zaagman WH, Mastebroeck HAK (1986) Adaptation of transient responses of a movement-sensitive neuron in the visual system of the blowfly Calliphora erythrocephala. Biol Cybern 54: 223-236

Eatock RA (2000) Adaptation in hair cells. Annu Rev Neurosci 23: 285-314

Egelhaaf M (2008) Fly vision: neural mechanisms of motion computation. Curr Biol 18: R339-R341

Egelhaaf M, Borst A (1989) Transient and steadystate response properties of movement detectors. J Opt Soc Am A 6: 116-127

Egelhaaf M, Borst A, Reichardt W (1989) Computational structure of a biological motion-detection system as revealed by local detector analysis in the fly's nervous system. J Opt Soc Am A 6: 1070-1087

Egelhaaf M, Grewe J, Karmeier K, Kern R, Kurtz R, Warzecha AK. (2005). Novel approaches to visual information processing in insects: case studies on neuronal computations in the blowfly. In: Christensen TA (ed) Methods in insect sensory neuroscience. CRC Press, Boca Raton, pp 185-212.

Egelhaaf M, Reichardt W (1987) Dynamic response properties of movement detectors: theoretical analysis and electrophysiological investigation in the visual system of the fly. Biol Cybern 56: 69-87

Fairhall AL, Lewen GD, Bialek W, de Ruyter van Steveninck RR (2001) Efficiency and ambiguity in an adaptive neural code. Nature 412: 787-792

Fettiplace R, Ricci AJ (2003) Adaptation in auditory hair cells. Curr Opin Neurobiol 13: 446-451
Harris RA, O'Carroll DC, Laughlin SB (1999) Adaptation and the temporal delay filter of fly motion detectors. Vision Res 39: 2603-2613

Harris RA, O'Carroll DC, Laughlin SB (2000) Contrast gain reduction in fly motion adaptation. Neuron 28: 595-606

Hausen K, Egelhaaf M. (1989). Neural mechanisms of visual course control in insects. In: Stavenga DG and Hardie RC (eds) Facets of vision. Springer, Berlin, Heidelberg, pp 391-424.

Heitwerth J, Kern R, van Hateren JH, Egelhaaf M (2005) Motion adaptation leads to parsimonious encoding of natural optic flow by blowfly motion vision system. J Neurophysiol 94: 1761-1769

Kalb J, Egelhaaf M, Kurtz R (2008a) Adaptation changes directional sensitivity in a visual motion-sensitive neuron of the fly. Vision Res 48: 1735-1742

Kalb J, Egelhaaf M, Kurtz R (2008b) Adaptation of velocity encoding in synaptically coupled neurons in the fly visual system. J Neurosci 28 : 9183-9193

Kern R, Lutterklas M, Petereit C, Lindemann JP, Egelhaaf M (2001) Neuronal processing of behaviourally generated optic flow: experiments and model simulations. Network: Comput Neural Syst 12: 351-369

Kern R, van Hateren JH, Michaelis C, Lindemann JP, Egelhaaf M (2005) Function of a fly motion-sensitive neuron matches eye movements during free flight. PLoS Biol 3: e171

Kohn A (2007) Visual adaptation: physiology, mechanisms, and functional benefits. J Neurophysiol 97: $3155-3164$

Krapp HG (2000) Neuronal matched filters for optic flow processing in flying insects. Int Rev Neurobiol 44: 93-120

Krapp HG, Hengstenberg B, Hengstenberg R (1998) Dendritic structure and receptive-field organization of optic flow processing interneurons in the fly. J Neurophysiol 79: 1902-1917

Krapp HG, Hengstenberg R (1996) Estimation of self-motion by optic flow processing in single visual interneurons. Nature 384: 463-466

Kurtz R (2007) Direction-selective adaptation in fly visual motion-sensitive neurons is generated by an intrinsic conductance-based mechanism. Neuroscience 146: 573-583

Kurtz R, Beckers U, Hundsdorfer B, Egelhaaf M (2009a) Mechanisms of after-hyperpolarization following activation of fly visual motion-sensitive neurons. Eur J Neurosci 30: 567-577 
Kurtz R, Dürr V, Egelhaaf M (2000) Dendritic calcium accumulation associated with directionselective adaptation in visual motion-sensitive neurons in vivo. J Neurophysiol 84: 1914-1923

Kurtz R, Egelhaaf M, Meyer HG, Kern R (2009b) Adaptation accentuates responses of fly motion-sensitive visual neurons to sudden stimulus changes. Proc Biol Sci 276: 3711-3719

Kurtz R, Kalb J, Spalthoff C (2008) Examination of fly motion vision by functional fluorescence techniques. Front Biosci 13: 3009-3021

Lappe M (2000) Computational mechanisms for optic flow analysis in primate cortex. Int Rev Neurobiol 44: 235-268

Laughlin SB (2001) Energy as a constraint on the coding and processing of sensory information. Curr Opin Neurobiol 11: 475-480

Liang P, Kern R, Egelhaaf M (2008) Motion adaptation enhances object-induced neural activity in three-dimensional virtual environment. J Neurosci 28: 11328-11332

Lindemann JP, Kern R, van Hateren JH, Ritter $\mathrm{H}$, Egelhaaf M (2005) On the computations analyzing natural optic flow: quantitative model analysis of the blowfly motion vision pathway. J Neurosci 25: 6435-6448

Maddess T, Laughlin SB (1985) Adaptation of the motion-sensitive neuron $\mathrm{H} 1$ is generated locally and governed by contrast frequency. Proc R Soc Lond B Biol Sci 228: 251-275

Matthews HR, Reisert J (2003) Calcium, the twofaced messenger of olfactory transduction and adaptation. Curr Opin Neurobiol 13: 469-475

Menini A (1999) Calcium signalling and regulation in olfactory neurons. Curr Opin Neurobiol 9: 419-426

Neri P, Laughlin SB (2005) Global versus local adaptation in fly motion-sensitive neurons. Proc Biol Sci 272: 2243-2249

Neri P (2007) Fast-scale adaptive changes of di- rectional tuning in fly tangential cells are explained by a static nonlinearity. J Exp Biol 210: 3199-3208

Nordström K, O'Carroll DC (2009) The motion aftereffect: local and global contributions to contrast sensitivity. Proc Biol Sci 276: 1545-1554

O'Carroll DC, Bidwell NJ, Laughlin SB (1996) Insect motion detectors matched to visual ecology. Nature 382: 63-66

O'Carroll DC, Laughlin SB, Bidwell NJ, Harris RA (1997) Spatio-temporal properties of motion detectors matched to low image velocities in hovering insects. Vision Res 37: 3427-3439

Reches A, Gutfreund Y (2008) Stimulus-specific adaptations in the gaze control system of the barn owl. J Neurosci 28: 1523-1533

Reisenman C, Haag J, Borst A (2003) Adaptation of response transients in fly motion vision. I: Experiments. Vision Res 43: 1293-1309

Spalthoff C, Egelhaaf M, Tinnefeld P, Kurtz R (2010) Localized direction selective responses in the dendrites of visual interneurons of the fly. BMC Biol 8: 36

Srinivasan MV, Dvorak DR (1979) The waterfall illusion in an insect visual system. Vision Res 19: 1435-1437

Srinivasan MV, Laughlin SB, Dubs A (1982) Predictive coding: a fresh view of inhibition in the retina. Proc R Soc Lond B Biol Sci 216: 427-459

Srinivasan MV, Poteser M, Kral K (1999) Motion detection in insect orientation and navigation. Vision Res 39: 2749-2766

Ulanovsky N, Las L, Nelken I (2003) Processing of low-probability sounds by cortical neurons. Nat Neurosci 6: 391-398

Wark B, Lundstrom BN, Fairhall A (2007) Sensory adaptation. Curr Opin Neurobiol 17: 423-429

Webb B (2002) Robots in invertebrate neuroscience. Nature 417: 359-363 